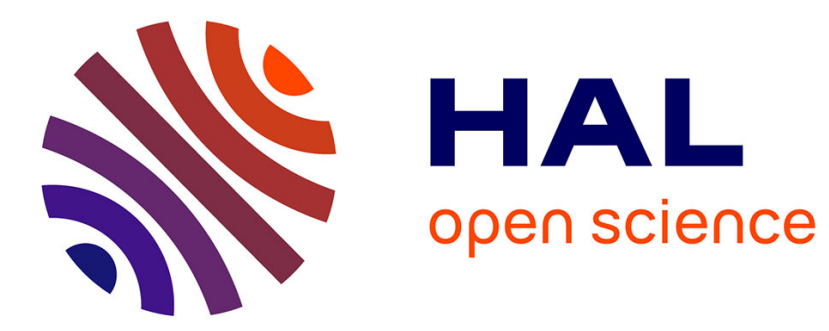

\title{
Low Frequency Model-Based Identification of Soft Impedance Faults in Cables
}

Andréa Cozza

\section{To cite this version:}

Andréa Cozza. Low Frequency Model-Based Identification of Soft Impedance Faults in Cables. IEEE Transactions on Instrumentation and Measurement, 2018, 10.1109/tim.2018.2879691 . hal-01895133

\section{HAL Id: hal-01895133 \\ https://hal-centralesupelec.archives-ouvertes.fr/hal-01895133}

Submitted on 14 Oct 2018

HAL is a multi-disciplinary open access archive for the deposit and dissemination of scientific research documents, whether they are published or not. The documents may come from teaching and research institutions in France or abroad, or from public or private research centers.
L'archive ouverte pluridisciplinaire HAL, est destinée au dépôt et à la diffusion de documents scientifiques de niveau recherche, publiés ou non, émanant des établissements d'enseignement et de recherche français ou étrangers, des laboratoires publics ou privés. 


\title{
Low Frequency Model-Based Identification of Soft Impedance Faults in Cables
}

\author{
Andrea Cozza
}

\begin{abstract}
Cables are subject to local impedance faults, or soft faults, e.g., following mechanical alterations. While their occurrence can be detected, no simple procedure exists for assessing whether a fault is critical and requires intervention. Previous work has demonstrated that the amplitude of echoes generated by time-domain reflectometry does not measure how severe such faults are, hindering attempts at introducing earlywarning schemes that could prevent these faults from eventually evolving into hard faults, i.e., open or short circuits. This paper introduces a model-based identification procedure that is capable of accurately inferring how severe an impedance modification along a cable is, together with its length. Its has the advantage of operating at lower frequencies than other more complex identification methods, while being intrinsically stable and welldefined since faults are identified and located in separate steps, without requiring non-linear regression techniques. The proposed method is also shown to remove the typical ambiguities found in the interpretation of time-domain reflectometry signals, by reinstating a single reflection peak in reflectograms. General feasibility conditions for the identification of impedance faults are discussed, proving that only sufficiently long faults can univocally be identified. The accuracy of the proposed method is tested against experimental results obtained for faults of increasing severity in coaxial cables, for which time-domain reflectometry is shown to yield accurate estimates only when testing over a bandwidth almost ten times wider.
\end{abstract}

Index Terms-Fault detection, identification, parameter inference, soft faults, cable testing, risk assessment.

\section{INTRODUCTION}

A LTHOUGH less often in the spotlight than wireless communications, cables are the backbone of communication infrastructures in a number of industrial settings whenever a highly reliable communication is required, from local-area networks in office buildings to transportation systems as airplanes and railway signaling, including critical applications such as control and security signals in power plants. The ability to detect any degradation in these cables is therefore fundamental and has led to the development of a number of measurement methods to detect and locate any fault that could compromise the propagation of signals and energy along cables.

The most prominent and widespread approach to fault detection and location is time-domain reflectometry (TDR). A large number of implementations has been introduced over the years [1]-[10], all sharing a similar idea: a test signal $p(t)$ is injected into a cable under test through a testing port and the output signal $e(t)$ is monitored for any significant echo, e.g., by comparing or cross-correlating it with $p(t)$.

Andrea Cozza is with GeePs, Group of electrical engineering - Paris UMR CNRS 8507, CentraleSupelec, Univ Paris-Sud, Sorbonne Universités, UPMC Univ Paris 06, 11 rue Joliot Curie, Plateau de Moulon 91192 Gif sur Yvette CEDEX.

Contact e-mail: andrea.cozza@ieee.org
A criterion (e.g., an amplitude threshold) is then applied in order to decide whether $e(t)$ contains significant anomalies that should be interpreted as a potential fault. The distance of the fault from the testing port is then inferred from the timeof-flight $\tau$ elapsed between the injection and the appearance of the anomaly. This approach is very effective as long as it is applied to the search of hard transitions, such as reflections from the end of a line or faults in the shape of short or open circuits, often referred to as hard faults. From the intensity of these echoes the reflectivity of the fault can be estimated and its nature identified, e.g., its impedance.

On the contrary, impedance (or soft) faults are local modifications found along a cable that can go from light chafing to partial removal of coatings or conductors. The difficulties in detecting them with TDR are well-known, basically due to their weak reflectivity [11]. Methods related to TDR specifically optimized to detect soft faults have been proposed [8], [9], [12], but they rely on the use of wide-band tests, exceeding several $\mathrm{GHz}$, that seem at odd with industrial settings and known frequency limitations from cables, in particular highfrequency attenuation.

Fundamental limitations to the use of TDR methods for soft-fault testing were highlighted in [13]. In particular, the amplitude of TDR echoes was shown not to represent an accurate estimator of how severe a fault is, as echoes also strongly depend on other parameters, e.g., the bandwidth of the test signal, $p(t)$, and the fault length $w$. In [14] it was shown that echoes generated by soft faults with a very different degree of severity can be practically identical, if tested at frequencies well below $f_{c} / 4$, with $f_{c}$ the fault characteristic frequency

$$
f_{c}=v / w=1 / T,
$$

where $T$ is the propagation delay needed for a signal to propagate across the fault, with a speed of propagation $v$. If tests are carried out at $f_{c} / 4$, an accurate estimate of a fault severity was shown to be possible. The problem is, for a $1 \mathrm{~cm}$ long fault in a PTFE-based coaxial cable, $f_{c} \simeq 20 \mathrm{GHz}$, thus requiring test signals to reach about $5 \mathrm{GHz}$ in order to identify the fault parameters, a requirement usually incompatible with industrial cables, e.g., because of high propagation losses.

Alternative methods have been recently introduced, based on the solution of an inverse problem relating reflection data and the characteristic impedance profile along a cable [15][17]. They also require wide-band tests: bandwidths from 5 up to $8 \mathrm{GHz}$ have been reported. Moreover, inverse-problem formulations are known for their tendency to be noise sensitive and may need regularization techniques.

There is a clear case for developing test methods capable of assessing whether a fault is critical enough to deserve 
attention, and costly human intervention, without relying on high-frequency data. This paper presents a methodology to solve this problem, by exploiting certain theoretical properties of impedance faults. A formal framework is introduced in Sec. II, where the accuracy and feasibility conditions for identification are discussed.

While the idea of inferring fault parameters using a modelbased approach has been previously considered in [12], [18], those papers did not explore the general properties and limitations about fault identification intrinsical to fault models. On the contrary, our proposal provides insight into the feasibility of fault identification, in particular the relationship existing between fault length and the minimum value of the test bandwidth $f_{M}$. Furthermore, the approach introduced in [12] is computationally intensive and critically based on a nonlinear regression procedure which is, by its very nature, illposed, requiring accurate initial guesses in order for it to be successful. This last issue also appeared to be a major limitation in [18].

Contrarily to these previous attempts, the propose method does not require the problems of identification and location to be solved simultaneously, avoiding to make the problem ill-posed because of its non-linear dependency to a fault parameters. A successful identification is shown to enable the synthesis of equalized TDR signals, yielding an accurate estimate of the positions of the fault extremities while removing ambiguities intrinsical to any standard TDR testing method. Experimental validation is presented in Sec. III, for several faults in coaxial cables, confirming theoretical predictions about feasibility conditions. In particular, faults are shown to be accurately identified at frequencies as low as $5 \%$ of $f_{c}$, thus strongly reducing the need for high-frequency testing.

These results are expected to be useful in designing effective and reliable early-warning monitoring systems based on robust criteria for automatic measurement-based decisions.

\section{MODEL-BASED FAULT IDENTIFICATION}

A soft fault can be schematically described as in Fig. 1, as a local change in the characteristic impedance of a cable, passing from its nominal value $Z_{o}$ to a local value $Z_{F}$, assumed to be constant over a length $w$. Transmission-line theory [19] allows to quantify this local impedance mismatch by defining a surge reflection coefficient $\Gamma_{o}$

$$
\Gamma_{o}=\frac{Z_{F}-Z_{o}}{Z_{F}+Z_{o}} .
$$

$\left|\Gamma_{o}\right|$ thus measures how severe a fault is and it is of practical importance to have access to this quantity. Unfortunately, $\Gamma_{o}$ cannot be directly measured from a testing port at one end of the cable. The reflection coefficient measured from this port is rather

$$
\Gamma_{\text {in }}(\nu)=\Gamma_{F}(\nu) \mathrm{e}^{-j 2 k d_{o}}
$$

where $d_{o}$ is the geometrical distance between the fault and the testing port, $\nu$ is the frequency, $k=2 \pi \nu / v$ the propagation constant of the cable and $v$ the speed of propagation. The fault reflectivity was found in [13] to be

$$
\Gamma_{F}(\nu)=\frac{2 \mathrm{j} \Gamma_{o} \mathrm{e}^{-\mathrm{j} k w} \sin (k w)}{1-\Gamma_{o}^{2} \mathrm{e}^{-\mathrm{j} 2 k w}}
$$

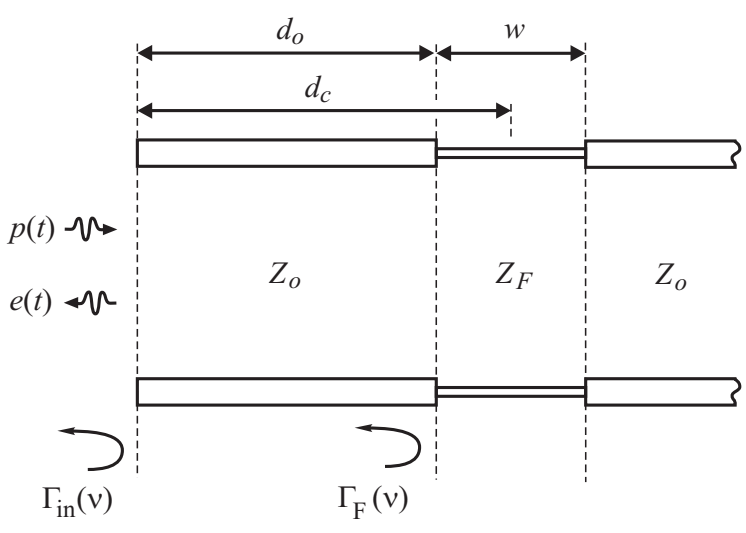

Figure 1. Double impedance-step representation of a local fault in a transmission line of characteristic impedance $Z_{o}$. The faulty section has a local characteristic impedance $Z_{F}$ and is centered at a distance $d_{c}$ from the testing port, spanning a length $w$ that starts at $d_{o}$. Its responds with reflection coefficients $\Gamma_{F}(\nu)$ and $\Gamma_{\text {in }}(\nu)$ when measured at the fault level or from a remote testing port, respectively.

with $w$ the length of the fault. Its accuracy was demonstrated in [13] together with its practical implications for fault testing and risk assessment.

Eq. (4) was shown in [13] to scale roughly with the frequency $\nu$ as long as $\nu \lesssim f_{o}$, where $f_{o}$ is the critical frequency of the fault

$$
f_{o}=\frac{f_{c}}{2 \pi} \frac{1-\Gamma_{o}^{2}}{1+\Gamma_{o}^{2}}
$$

which, since in most practical configurations $\left|\Gamma_{o}\right|<0.5$, can be approximated by

$$
f_{o} \simeq \frac{f_{c}}{2 \pi}\left(1-\Gamma_{o}^{2}\right)^{2}
$$

with an error at worst equal to $6.7 \%$, reduced to $0.8 \%$ for $\left|\Gamma_{o}\right|<0.3$.

Fig. 2 presents the reflectivity $\left|\Gamma_{F}(\nu)\right|$ from an impedance fault as in Fig. 1, for several values of severity $\left|\Gamma_{o}\right|$. For $\nu<f_{o},\left|\Gamma_{F}(\nu)\right|$ is indeed mostly proportional to $\nu$, but clear differences are apparent according to the severity of the fault, $\left|\Gamma_{o}\right|$, in terms of an increasing curvature of $\left|\Gamma_{F}(\nu)\right|$, as $\nu / f_{o} \rightarrow 1$.

Fig. 2 suggests the possibility of linking this curvature to the severity of a fault, an idea developed in Sec. II-A, by introducing a formal framework for the identification of fault parameters, inferring their value from regression of measurement results onto a model. The accuracy and feasibility of this approach are discussed in Sec. II-B, while the opportunities to simplify fault location, enabled by fault identification, are discussed in Sec. II-C.

It is of practical interest that the input reflection coefficient $\Gamma_{\text {in }}(\nu)$ measured from one end of a cable has $\left|\Gamma_{\text {in }}(\nu)\right| \simeq$ $\left|\Gamma_{F}(\nu)\right|$, as long as a cable is tested at frequencies at which it is weakly dissipative. This observation implies that any identification method based only on the amplitude of a fault reflectivity would not be affected by phase dispersion in signals propagating through the cable, when testing a cable at frequencies higher than its normal operating conditions. Clearly, 


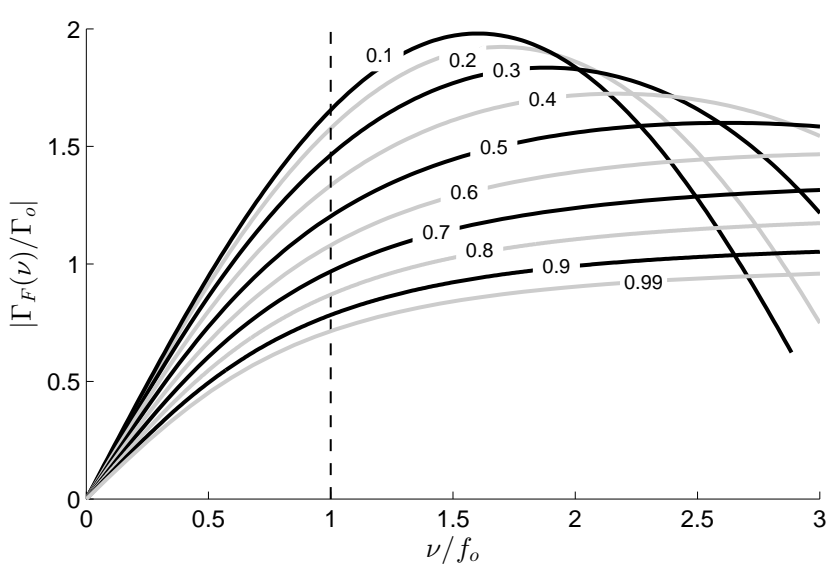

Figure 2. Normalized fault reflectivity predicted by (4) as a function of the frequency $\nu$ and the fault severity $\left|\Gamma_{o}\right|$, shown on each curve. Frequency is normalized to $f_{o}$, yielding results independent from the fault length $w$. For $\nu / f_{o}<1,\left|\Gamma_{F}(\nu)\right|$ roughly scales with the frequency.

propagation losses can be compensated from a preliminary calibration on healthy cables, but this case is not considered here. Assuming $\left|\Gamma_{\text {in }}(\nu)\right| \simeq\left|\Gamma_{F}(\nu)\right|$ in the following, the fault location can be considered in a second step, as the propagation modeled by the exponential in (3) has no effect.

The proposed procedure avoids non-linear regressions by proceeding first to identify the fault parameters using a linear regression and exploits these results for locating the fault in a subsequent step. This approach is computationally frugal, and makes the design of testing methods possible, since the accuracy of the results can be predicted. Finally, it answers the need for low-frequency testing, while handing good estimates, without requiring ultra-wide bandwidth signals, as done in previous works cited in Sec. I.

\section{A. Fault identification}

The relationship between the curvature of $\left|\Gamma_{F}(\nu)\right|$ and the fault parameters can be established by expanding $\left|\Gamma_{F}(\nu)\right|$, as defined in (4), into a power series, in order to factorize the relative contributions of frequency and fault parameters. Proceeding to a Taylor expansion for $\nu \rightarrow 0$ yields

$$
\begin{aligned}
\left|\Gamma_{F}(\nu)\right| & =\left|\Gamma_{o}\right| \sum_{n=0}^{\infty} b_{n}(\nu)= \\
& =\left|\Gamma_{o}\right| \sum_{n=0}^{\infty} w_{n}\left(\Gamma_{o}\right)\left(\frac{\nu}{f_{o}}\right)^{2 n+1} \\
& =\left|\Gamma_{o}\right| \sum_{n=0}^{\infty} \frac{(-1)^{n} a_{n}\left(\Gamma_{o}\right)}{\left(1+\Gamma_{o}^{2}\right)^{2 n+1}}\left(\frac{\nu}{f_{o}}\right)^{2 n+1}
\end{aligned}
$$

where $\left\{a_{n}\left(\Gamma_{o}\right)\right\}$ are polynomials in the parameter $\Gamma_{o}$

$$
\begin{aligned}
a_{0} & =2 \\
a_{1} & =\left(1+10 \Gamma_{o}^{2}+\Gamma_{o}^{4}\right) / 3 \\
a_{2} & =\left(1+116 \Gamma_{o}^{2}+486 \Gamma_{o}^{4}+116 \Gamma_{o}^{6}+\Gamma_{o}^{8}\right) / 60 \\
a_{3} & =\left(1+1086 \Gamma_{o}^{2}+20847 \Gamma_{o}^{4}+56932 \Gamma_{o}^{6}+\right. \\
& \left.+20847 \Gamma_{o}^{8}+1086 \Gamma_{o}^{10}+\Gamma_{o}^{12}\right) / 2520 .
\end{aligned}
$$

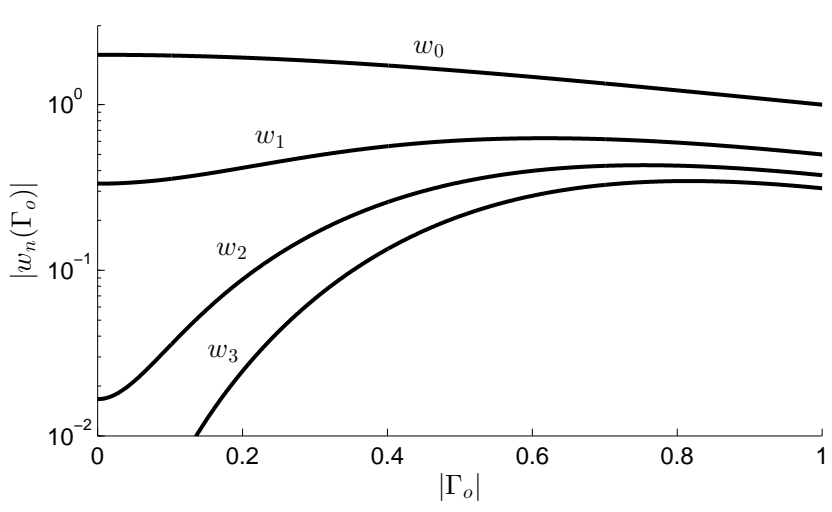

Figure 3. Weights $w_{n}\left(\Gamma_{o}\right)$ of the Taylor expansion (7) of the fault reflectivity $\left|\Gamma_{F}(\nu)\right|$, as functions of the fault severity $\left|\Gamma_{o}\right|$.

The rationale for expanding $\left|\Gamma_{F}(\nu)\right|$ in the variable $\nu / f_{o}$ is twofold. First, it provides simpler expressions where the fault extension $w$ has no effect on the polynomial coefficients $\left\{w_{n}\left(\Gamma_{o}\right)\right\}$. Second, as proven in Sec. II-B, the ability of $f_{o}$ to identify the frequency range where the fault reflectivity starts to deviate from its low frequency regime, thus confirming its role as an important fault parameter.

Fig. 3 shows how the amplitudes of the expansion coefficients $\left\{w_{n}\left(\Gamma_{o}\right)\right\}$ evolve with the fault severity $\Gamma_{o}$, with the first two terms of the series providing the main contributions for $\left|\Gamma_{o}\right|<0.4$. These results confirm that deviation from linearity in $\nu$ are tightly related to the fault severity $\left|\Gamma_{o}\right|$.

Fig. 4(a) completes this picture by showing that the contribution from $b_{1}(\nu)$ is about $20 \%$ of $b_{0}(\nu)$ if faults with $\left|\Gamma_{o}\right| \leq 0.3$ are involved, as long as tests are carried out for $\nu<f_{o}$. As a result, measurements taken at frequencies $\nu \ll f_{o}$ could be expected not to be reliable for the identification of a fault, since in this case (3) can be approximated as

$$
\Gamma_{\mathrm{in}}(\nu) \simeq \frac{2 \mathrm{j} \nu}{v} \Gamma_{o} w \mathrm{e}^{-j 2 k d_{c}},
$$

where the roles of $\Gamma_{o}$ and $w$ are hardly distinguishable. Fig. 4(b) shows that $b_{2}(\nu)$ starts having an impact as $\left|\Gamma_{o}\right| \gtrsim 0.3$, i.e., for rather severe impedance faults (cf. the examples in Sec. III) at relatively high frequencies.

As also shown in Fig. 3, terms for $n \geq 2$ are much more sensitive to the fault severity $\left|\Gamma_{o}\right|$ than lower-order terms. It would therefore be tempting to consider exploiting this property in order to estimate $\left|\Gamma_{o}\right|$. The problem with such a proposal is the minor contribution of $b_{n}(\nu)$ for $n \geq 2$, for soft faults and/or tests carried out below $f_{o}$, which leaves them more exposed to noise.

The fault parameters can now be identified by first approximating measured data by means of a polynomial of order $N$ (here the number of terms) and degree $2 N+1$

$$
\left|\Gamma_{F}(\nu)\right| \simeq \sum_{n=0}^{N} p_{n} \nu^{2 n+1},
$$

obtaining the polynomial coefficients $\left\{p_{n}\right\}$, e.g., by means of a least-square regression. A detailed analysis about the choice of 

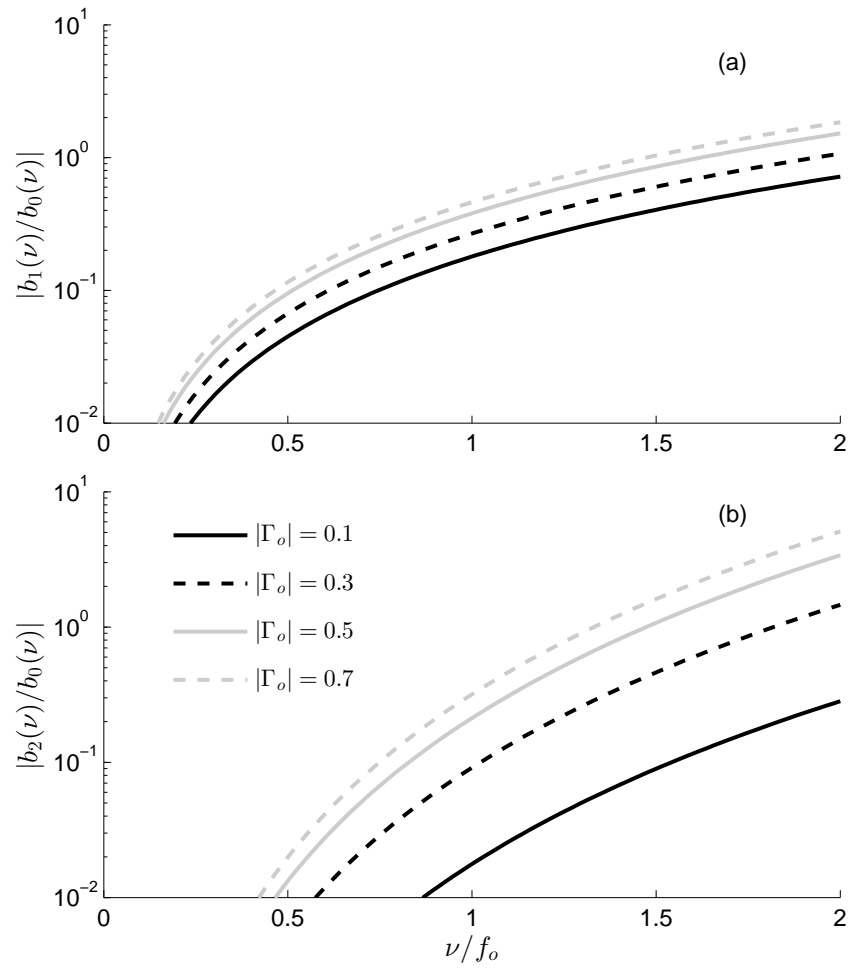

Figure 4. Partial contributions to (7) of higher-order $\left\{b_{n}(\nu)\right\}$ relative to the linear term $b_{0}(\nu)$, as a function of the frequency and the fault severity $\left|\Gamma_{o}\right|$.

$N$ and its impact on the accuracy of the procedure is presented in Sec. II-B. Enforcing the equivalence between (7) and (10),

$$
p_{n}=(2 \pi T)^{2 n+1}(-1)^{n} \frac{\left|\Gamma_{o}\right| a_{n}\left(\Gamma_{o}\right)}{\left(1-\Gamma_{o}^{2}\right)^{2 n+1}} \quad \forall n \leq N,
$$

would result in an estimate of $\left|\Gamma_{o}\right|$ and $w$. In practice, this direct approach is not the simplest option, as it would require to solve an over-determined non-linear system of $N+1$ equations in the two unknowns $\Gamma_{o}$ and $w$. Moreover, as already discussed, higher-order polynomial contributions would be more affected by noise.

It is possible to directly obtain estimators by focusing on the first two terms in (7). We stress the fact that using only $p_{0}$ and $p_{1}$ does not mean that $N$ needs to be set to one in (10), as discussed in Sec. II-B. Now, enforcing (11) on $n \leq 1$

$$
\begin{aligned}
p_{0} & =2 \pi T \frac{2\left|\Gamma_{o}\right|}{1-\Gamma_{o}^{2}} \\
-p_{1} & =(2 \pi T)^{3} \frac{\left|\Gamma_{o}\right|\left(1+10 \Gamma_{o}^{2}+\Gamma_{o}^{4}\right) / 3}{\left(1-\Gamma_{o}^{2}\right)^{3}}
\end{aligned}
$$

straightforwardly yields an estimate of $\left|\Gamma_{o}\right|$, by setting

$$
s=-\frac{p_{1}}{p_{0}^{3}}=\frac{1+10 \Gamma_{o}^{2}+\Gamma_{o}^{4}}{24 \Gamma_{o}^{2}},
$$

which yields the estimator

$$
\hat{\Gamma}_{o}^{2}=-5+12 s-2 \sqrt{6} \sqrt{1-5 s+6 s^{2}} .
$$

Most settings call for $\left|\Gamma_{o}\right|<0.5$, for which $\left|\Gamma_{o}\right|^{4} \simeq 0$ in (13), thus yielding

$$
\left|\hat{\Gamma}_{o}\right|=1 / \sqrt{-24 p_{1} / p_{0}^{3}-10}
$$
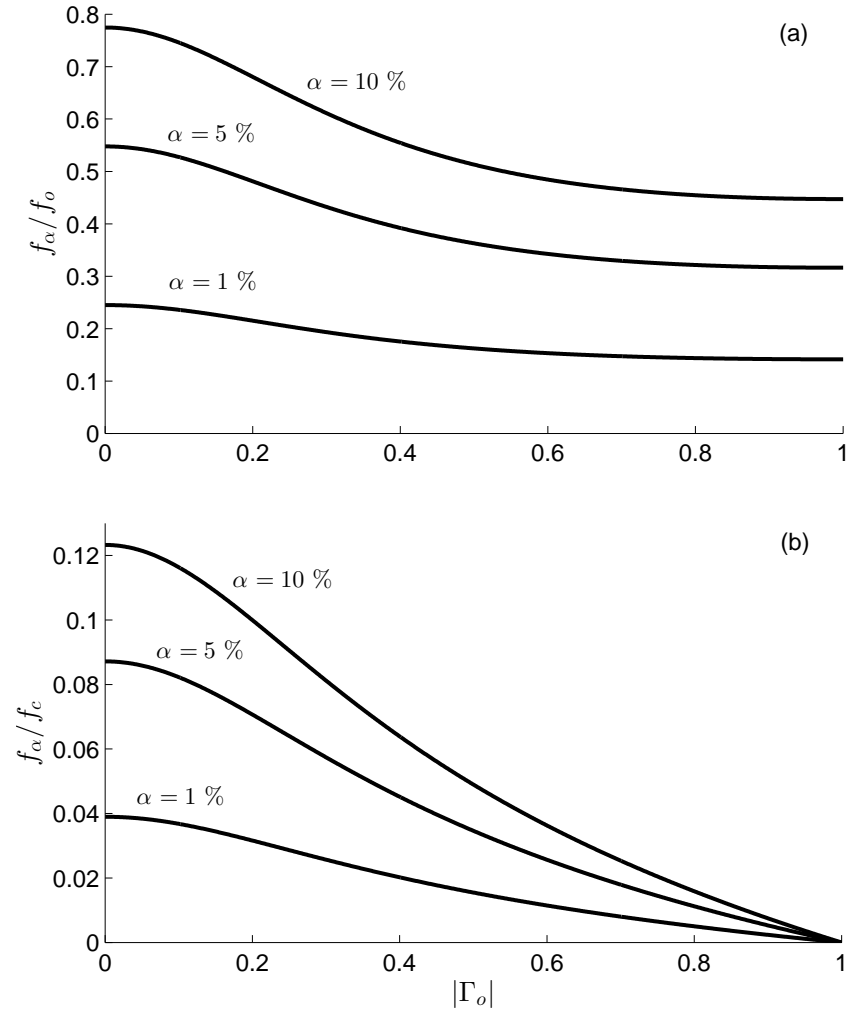

Figure 5. Frequency $f_{\alpha}$ for which $\left|b_{1}\left(f_{\alpha}\right) / b_{0}\left(f_{\alpha}\right)\right|=\alpha$, expressed as either a fraction of (a) the critical frequency $f_{o}$ or (b) the characteristic frequency $f_{c}$ of the fault. The value of $\alpha$ is defined by the degree of accuracy of available data.

The fault length estimator $\hat{w}$ is then found from (12a), recalling that $w=T v$

$$
\hat{w}=p_{0} v \frac{1-\Gamma_{o}^{2}}{4 \pi\left|\Gamma_{o}\right|}
$$

\section{B. Polynomial regression and identification accuracy}

The fault parameter estimators (14)-(16) require an accurate estimate of the polynomial coefficients $p_{0}$ and $p_{1}$. The previous section has shown that in low-frequency testing, i.e., well below $f_{o}$, the reflectivity of an impedance fault is mostly proportional to the frequency, so that any identification attempt is bound to fail unless the contribution of at least the first higher-degree term $b_{1}(\nu)$ can be measured, which can be challenging in case of data affected by noise. More generally, including higher-degree terms may result in an overfitting of the data samples, with (10) reproducing noise contributions rather than operating as a regression procedure, which would otherwise be expected to be robust to noise [20].

It is therefore important to understand what is the minimum value of test bandwidth $f_{M}$ to be considered in order for the proposed fault identification procedure to be applied successfully, e.g., by requiring that the non-linear contribution $\left|b_{1}(\nu)\right|$ be at least a fraction $\alpha$ of $\left|b_{0}(\nu)\right|$. The frequency $f_{\alpha}$ for which $\left|b_{1}(\nu) / b_{0}(\nu)\right| \geq \alpha, \forall \nu>f_{\alpha}$ is straightforwardly found from (7) to be

$$
f_{\alpha} / f_{o}=\sqrt{6 \alpha} \frac{1+\Gamma_{o}^{2}}{\sqrt{1+10 \Gamma_{o}^{2}+\Gamma_{o}^{4}}} .
$$


Its dependence on the fault severity $\left|\Gamma_{o}\right|$ is shown in Fig. 5(a), for several values of $\alpha$. These results show that $f_{o}$ provides an effective estimator of the frequency at which a fault response deviates from a linear slope, since $f_{\alpha} / f_{o}$ is not strongly dependent on the value of $\left|\Gamma_{o}\right|$, especially for $\alpha<10 \%$.

Conversely, expressing (17) in terms of the characteristic frequency $f_{c}$ yields

$$
f_{\alpha} / f_{c}=\frac{\sqrt{6 \alpha}}{2 \pi} \frac{1-\Gamma_{o}^{2}}{\sqrt{1+10 \Gamma_{o}^{2}+\Gamma_{o}^{4}}},
$$

which is shown in Fig. 5(b) to be way more sensitive to $\left|\Gamma_{o}\right|$. These observations justify the choice of using $f_{o}$ as a reference in the description of fault models as (7). Eq. (5) provides direct insight in the identification process, as it suggests that more severe faults can be identified at lower frequencies, as $f_{o}$ decreases for a higher severity $\left|\Gamma_{o}\right|$.

The value of $\alpha$ depends on the uncertainty expected from measurements, in particular, but not exclusively, from additive noise. Assuming an additive white Gaussian noise with standard deviation $\sigma_{n}$, requiring that $b_{1}\left(f_{\alpha}\right)>2 \sigma_{n}$ results in

$$
\alpha>\frac{2 \sigma_{n}}{\left|\Gamma_{F}\left(f_{\alpha}\right)\right|}=\frac{2}{\sqrt{\mathrm{SNR}}}
$$

with SNR the signal-to-noise ratio of the measured reflection coefficient $\left|\Gamma_{F}\right|$ at $f_{\alpha}$.

A $\alpha>0$ acknowledges the fact that there is no need to extend the polynomial fit in (10) by including higher-order terms whose contribution could be smaller than uncertainty. E.g., from the results presented in Sec. III, $\alpha$ was inferred to be about $2 \%$, requiring $f_{M}>f_{o} / 20$, approximatively. Fig. 5 requires that $f_{o}$ be known, a problem addressed at the end of this section.

Thanks to (1), the condition $f_{M}>f_{\alpha}$ can be translated as

$$
w \geq \frac{v}{f_{M}} \frac{f_{\alpha}}{f_{c}},
$$

which is a necessary condition to ensure the feasibility of impedance fault identification, in terms of a fault length and the chosen test bandwidth. E.g., assuming a PTFE-based coaxial cable, tested with signals of bandwidth $f_{M}=300$ $\mathrm{MHz}$, with an expected $\alpha=5 \%$, corresponding to $f_{\alpha} / f_{c} \simeq$ 0.08 , then only faults at least $5 \mathrm{~cm}$ long could be identified unambiguously. A simpler but more conservative criterion was introduced in [14],

$$
w \geq \frac{v}{4 f_{M}}
$$

based on the requirement of allowing direct estimates to be drawn from frequency-domain data as recalled in Sec. III. TDR testing would rather require $f_{M}>f_{c} / 2$ (cf. Sec. III-B), i.e., $f_{M} \gtrsim 2 \mathrm{GHz}$ for the proper identification of a $5 \mathrm{~cm}$ fault.

The condition (20) thus allows to dramatically reduce the minimum test bandwidth needed for fault identification, by about a factor 3 for the above example, and a factor 6 with respect to standard TDR. This latter reduction will be shown to be closer to a factor 12 for the experimental results shown in Sec. III, confirming that the procedure here proposed
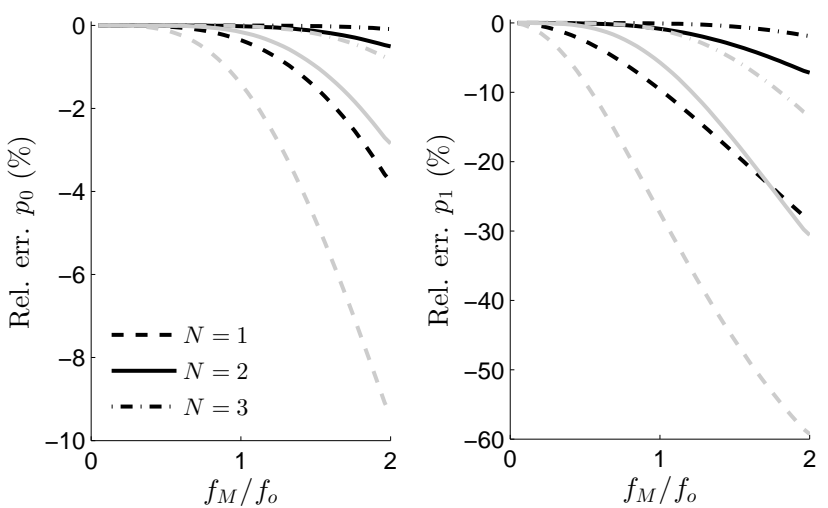

Figure 6. Relative error in the expansion parameters $p_{0}$ and $p_{1}$ estimated from (10) as a function of the order $N$ of the polynomial and the test bandwidth $f_{M}$. Results refer to a fault with $\left|\Gamma_{o}\right|=0.1$ (black curves) and $\left|\Gamma_{o}\right|=0.3$ (gray curves).

can provide accurate fault identification while testing at low frequency.

If $\alpha>0$ pushes to restrain from using higher-order polynomials, it is still necessary to choose $N$ large enough to reproduce the fault reflectivity accurately. As $f_{M} / f_{o}$ and $\left|\Gamma_{o}\right|$ increase, a higher-order polynomial is required in order to properly reproduce the fault reflectivity $\left|\Gamma_{F}(\nu)\right|$, and thus yield accurate estimates of $p_{0}$ and $p_{1}$, upon which identification formulas (15) and (16) depend. This issue should therefore be regarded as a source of systematic errors, i.e., present also in case of noiseless data.

The question of how $N$ should be chosen can be answered by applying the least-square fit (10) to (4), over a given frequency range $f_{M}$, for a specific value of $\left|\Gamma_{o}\right|$ and $N$. By comparing the fit parameters $\left\{p_{n}\right\}$ to those expected from theory, i.e., $\left\{w_{n} / f_{o}^{2 n+1}\right\}$ as from (7), the best choice of $N$ can be studied, in order to control this source of systematic errors.

Fig. 6 presents the results of this analysis for the case of a relatively light fault with $\left|\Gamma_{o}\right|=0.1$. As long as $f_{M} / f_{o} \leq 1$, $p_{0}$ can be expected to be within less than $1 \%$ of its expected value, with a $10 \%$ error on $p_{1}$ for $N=1$, i.e., for the simplest regression model possible. In this case, recalling (15), $\left|\Gamma_{o}\right|$ estimated from (15) would present a systematic error smaller of approximatively $5 \%$. Much better accuracy can be achieved for $N>1$, but this possibility needs to be explored bearing in mind that higher-order terms can be estimated only if their contribution is larger than the measurement uncertainty. The risk would otherwise be to overfit data fluctuations. For $f_{M}>f_{o}$ the accuracy quickly worsens and choosing $N>1$ is necessary, as confirmed by Sec. III. When more severe faults are considered, $N>1$ might be needed even for $f_{M}<f_{o}$, as in Fig. 6 for $\left|\Gamma_{o}\right|=0.3$, since the estimate error for $p_{0}$ and $p_{1}$ is about twice that for $\left|\Gamma_{o}\right|=0.1$. This conclusion is explained by the no longer negligible contribution from $b_{2}(\nu)$, thus requiring $N \geq 2$.

Fig. 7 show how these systematic errors on $p_{0}$ and $p_{1}$ translate into biases in fault parameter estimates.

A simple criterion that can help in deciding about the 

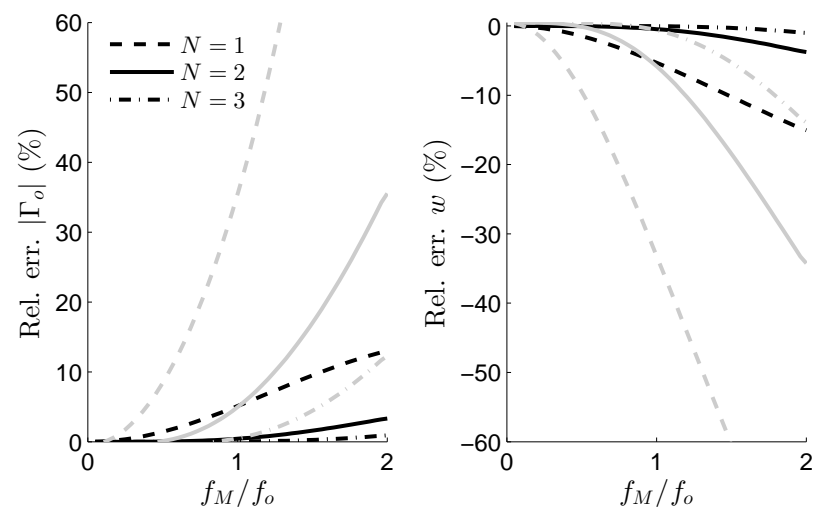

Figure 7. Relative error in fault parameters $\left|\Gamma_{o}\right|$ and $w$ estimated from (10) as a function of the order $N$ of the polynomial and the maximum tested frequency $f_{M}$. Results refer to a fault with $\left|\Gamma_{o}\right|=0.1$ (black curves) and $\left|\Gamma_{o}\right|=0.3$ (gray curves).

reliability of estimators of the fault parameters would call for the fault critical frequency $f_{o}$ to be known, hence allowing to verify the value of $f_{M} / f_{o}$. From (12)

$$
-\frac{p_{0}}{p_{1}}=6 f_{o}^{2} \frac{\left(1+\Gamma_{o}^{2}\right)^{2}}{1+10 \Gamma_{o}^{2}+\Gamma_{o}^{4}} \simeq 6 f_{o}^{2}\left(1-8 \Gamma_{o}^{2}\right)
$$

which, for $\Gamma_{o}^{2} \ll 1$ yields

$$
\hat{f}_{o} \simeq \sqrt{-p_{0} / 6 p_{1}}
$$

The above estimator is expected to underestimates $f_{o}$ by less than $21 \%$ as long as $\left|\Gamma_{o}\right|<0.3$. The experimental validation presented in Sec. III holds (23) accurate for $f_{M}>f_{o} / 3$.

\section{Locating the fault region: equalized TDR}

Apart from the identification of an impedance fault, TDRbased approaches present another issue: to the best of our knowledge, there is no clear procedure for the interpretation of the echo generated by such faults. Fig. 8(a) shows a typical echo from this class of faults, observed in one of the experiments described in Sec. III. Recalling that TDR echoes in presence of hard faults are expected to be proportional to the test signal, one could be tempted to think that the peaks in the echo represent the partial reflections from the two ends of the region spanned by the impedance fault. The inaccuracy of this interpretation was discussed in [13] and it is especially troublesome since these peaks only depend on the bandwidth of the test signal.

Instead of attempting any interpretation based on standard TDR, once the fault parameters $\left|\Gamma_{o}\right|$ and $w$ are identified, (4) can be used in order to remove any ambiguity in the interpretation of fault echoes. For a measured TDR echo $e(t)$, its Fourier transform reads

$$
E(\nu)=P(\nu) \Gamma_{\text {in }}(\nu)=P(\nu) \Gamma_{F}(\nu) \mathrm{e}^{-2 \mathrm{j} k d_{o}}
$$

where $P(\nu)$ is the Fourier spectrum of the test signal $p(t)$ and $d_{o}$ is the distance between the test port and the left end of the fault, as in Fig. 1.

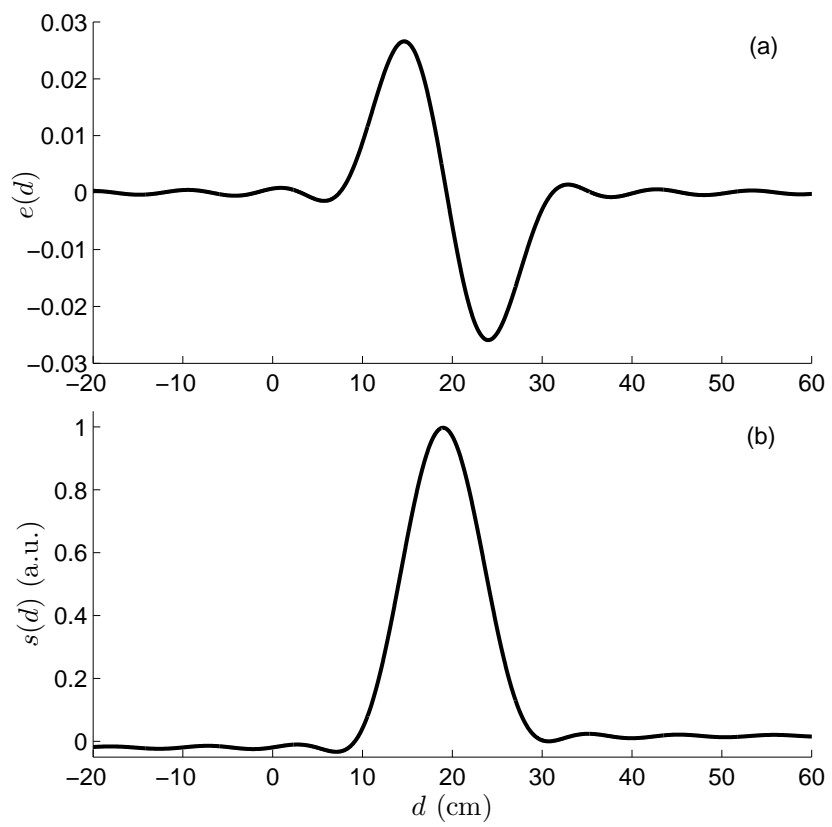

Figure 8. A typical example of TDR echo for an impedance fault, displaying the distinctive monocycle pulse shape (a) and the equalized TDR echo (b), unambiguously indicating the beginning of the fault region. Refer to Sec. III for details.

A signal $s(t)$ can be synthesized by inverse transforming the equalized spectrum

$$
S(\nu)=\frac{E(\nu)}{\hat{\Gamma}_{F}(\nu)}=\frac{\Gamma_{F}(\nu)}{\hat{\Gamma}_{F}(\nu)} P(\nu) \mathrm{e}^{-2 \mathrm{j} k d_{o}},
$$

with $\hat{\Gamma}_{F}(\nu)$ the fault reflectivity obtained by evaluating (4) with the estimated fault parameters, recalling that $\Gamma_{o}$ is only known by its modulus. Therefore, for an accurate identification

$$
S(\nu) \simeq \operatorname{sign}\left(\Gamma_{o}\right) P(\nu) \mathrm{e}^{-2 \mathrm{j} k d_{o}},
$$

which reproduces conditions similar to those expected for hard faults, since

$$
s(t) \simeq \operatorname{sign}\left(\Gamma_{o}\right) p(t-\tau),
$$

where $\tau=2 d_{o} / v$. Hence, $s(t)$ will present a single peak, reached over the left end of the fault region, at a distance $d_{o}$ from the test port, also correcting the $w / 2$ shift appearing in (4). Fig. 8(b) shows how this procedure operates on the original TDR echo in Fig. 8(a). The amplitude of the equalized echo does no longer bear any information about the fault reflectivity, since (27) effectively removes all fault-dependent quantities, but for its position. The effectiveness and accuracy of this procedure is demonstrated in Sec. III.

In case $\left|\Gamma_{o}\right| \ll 1$, it can be expected that the distance from the center of the fault will be identified by the time at which $e(t)$ crosses zero between the two peaks in the TDR echo doublet. This approach makes sense as long as a perfectly symmetric $e(t)$ can be expected. It is important to notice that the equalization procedure is more general, as it would work even in case of critically severe faults, where a TDR echo $e(t)$ may display asymmetric features, as discussed in [13]. 


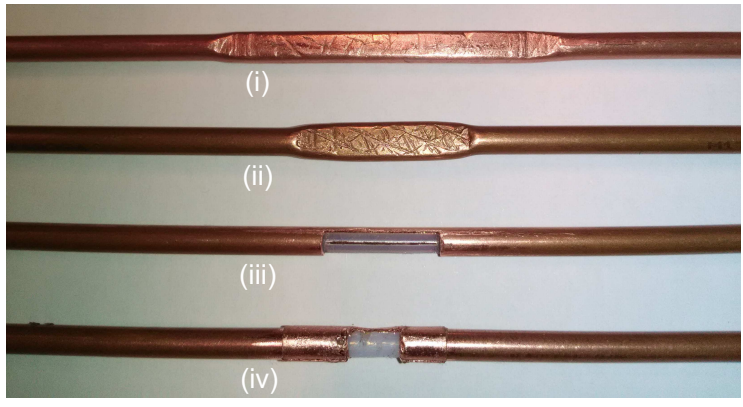

Figure 9. The four cable faults tested, sorted from the least (i) to the most severe (iv).

In case $f_{M}<f_{o},\left|\Gamma_{F}(\nu)\right|$ would increasingly appear as scaling linearly with the frequency $\nu$ and the identification procedure in Sec. II-B might no longer be reliable. This not withstanding, (25) can still be applied, as the polynomial fit can correct the frequency dispersion introduced by $\left|\Gamma_{F}(\nu)\right|$, even though the absence of parameter estimates does no longer allow to correct the delay term $w / 2$. The resulting signal

$$
s_{e}(t) \simeq \operatorname{sign}\left(\Gamma_{o}\right) p(t-\tau-T),
$$

with $T$ defined in (1), will share the same property of proportionality to $p(t)$ as $s(t)$, but will now reach its peak over the fault center, at a distance $d_{c}$.

\section{EXPERIMENTAL RESULTS}

The proposed procedure was put to test with the four $30 \mathrm{~cm}$ long semi-rigid coaxial cables shown in Fig. 9. These faults range from a crushed to an almost severed outer conductor. Their lengths, varying from slightly less than $1 \mathrm{~cm}$ to more than $4 \mathrm{~cm}$, were designed in order to present very similar responses when tested below $1 \mathrm{GHz}$, as discussed in [14]. The four cables are based on a PTFE (polytetrafluoroethylene) dielectric and have a $50 \Omega$ characteristic impedance. Their average phase velocity over a $6 \mathrm{GHz}$ bandwidth was estimated on an undamaged cable from transmission delays, resulting in $v \simeq 2.18 \times 10^{8} \mathrm{~m} / \mathrm{s}$, with negligible dispersion up to 6 GHz. The velocity $v$ is a quantity of interest not only for converting time delays into distances, but also for estimating the fault length $w$. It should be noticed that the same velocity is assumed for the undamaged cable as well as for the faulty portions, as apparent in (4).

The reflectivity of each fault was measured using the setup in Fig. 10, where a vector network analyzer (VNA), model R\&S ZVB8, measured the input reflectivity $\Gamma_{\text {in }}(\nu)$ of the cables over the frequency range $100 \mathrm{kHz}$ to $6 \mathrm{GHz}$. According to the VNA manual, a maximum relative uncertainty of $5 \%$ should be expected for measurements involving a reflectivity $<0.1$, with a minimum absolute uncertainty equal to 0.03 .

Each cable was terminated by a $50 \Omega$ impedance, in order to reduce reflections from its far end. The rationale for this choice was that given the short length of the cable and the close proximity of its far end to the fault (less than $15 \mathrm{~cm}$ ), echoes from the cable end would superpose to those from the faults for most choices of the test bandwidth $f_{M}$. In most practical settings, for long cables of several tens meters or even

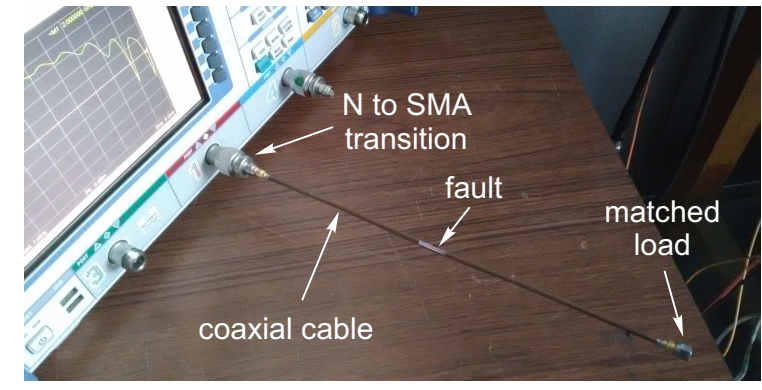

Figure 10. The experimental setup with the vector network analyzer used for characterizing the cables.

more, such occurrence would be unlikely. The use of short rigid coaxial cables was intended to avoid using longer ones that would have not allowed to apply controlled impedance modifications, as those in Fig. 9, because they would have needed to be pliable in order to be rolled and thus less easily machineable. For similar reasons, the frequency range of test was in the $\mathrm{GHz}$ range because of the limited extent of the faults, again a choice dictated by the use of a compact validation setup.

Given the weak intensity of fault reflectivity, even small reflections from the $\mathrm{N}$ to SMA connector in Fig. 10 can be an issue. Careful calibration after the connector was applied using the full 1-port correction procedure available for this VNA. Fault echoes were isolated from residual reflections from the $50 \Omega$ far-end load by means of time gating. This approach should be applied systematically in cable testing, in order to allow frequency-domain procedures as the one here proposed, starting from data obtained from TDR techniques. Alternatively, wavelet expansions could be used, as they also allow effective data denoising while capturing a signal over a limited time interval. This kind of approach allows processing data presenting multiple echoes of interest, by processing them on a one-by-one basis.

Fig. 11 shows the reflectivity of the faults thus characterized. The wide frequency range allowed to access the high-frequency behavior of the faults, in order to provide accurate identification that served as a reference throughout the validation of the low-frequency identification procedure.

From $\left|\Gamma_{F}(\nu)\right|$ in Fig. 11 it is possible to estimate the faults severity $\left|\Gamma_{o}\right|$ by applying the procedure introduced in [14]. $\left|\Gamma_{F}(\nu)\right|$ reaches its first maximum $\Gamma_{M}$ at the frequency $\nu^{\star}=$ $f_{c} / 4$, with (4) giving

$$
\Gamma_{M}=\left|\Gamma_{F}\left(f_{c} / 4\right)\right|=\frac{2\left|\Gamma_{o}\right|}{1+\Gamma_{o}^{2}} .
$$

Since all faults in Fig. 9 are fundamentally local impedance changes that do not introduce any measurable losses, $\Gamma_{o} \in \mathbb{R}$, hence inverting (29) yields

$$
\left|\Gamma_{o}\right|=\frac{1-\sqrt{1-\Gamma_{M}^{2}}}{\Gamma_{M}} \simeq \frac{\Gamma_{M}}{2},
$$

while the fault length can be estimated as $w_{e}=v / 4 \nu^{\star}$. A direct fit was applied for fault (iv), as no local maximum is reached. Since the propagation speed along the fault is 
Table I

FAULT PARAMETERS: $d_{o}, d_{c}$ AND $w$ ARE GEOMETRICAL DISTANCES MEASURED ON THE FAULTS WITH AN EXPECTED UNCERTAINTY UNIFORMLY DISTRIBUTED SPANNING A \pm 0.5 MM RANGE. $w_{e}$ AND $\left|\Gamma_{o}\right|$ ARE THE FAULTS PARAMETERS ESTIMATED ACCORDING TO THE PROCEDURE RECALLED IN SEC. III. THEORETICAL RESULTS PREDICTED BY (4) FOR THESE SETS OF PARAMETERS ARE SHOWN IN FIG. 11. THE FAULTS CRITICAL AND CHARACTERISTIC FREQUENCIES, RESPECTIVELY $f_{o}$ AND $f_{c}$, WERE COMPUTED FROM $w_{e}$ AND $\left|\Gamma_{o}\right|$.

\begin{tabular}{|c|c|c|c|c|c|c|c|}
\hline \multirow{2}{*}{ Faults } & $d_{o}$ & $d_{c}$ & $w$ & $w_{e}$ & $\left|\Gamma_{o}\right|$ & $f_{o}$ & $f_{c}$ \\
\cline { 2 - 8 } & $(\mathrm{cm})$ & $(\mathrm{cm})$ & $(\mathrm{mm})$ & $(\mathrm{mm})$ & & $(\mathrm{GHz})$ & $(\mathrm{GHz})$ \\
\hline \hline (i) & 13.9 & 16.1 & 44 & 44 & 0.042 & 0.79 & 5.0 \\
(ii) & 15.0 & 16.0 & 21 & 20 & 0.091 & 1.7 & 11 \\
(iii) & 15.4 & 16.0 & 14 & 14 & 0.13 & 2.4 & 16 \\
(iv) & 16.0 & 16.4 & 6 & 7 & 0.22 & 4.4 & 30 \\
\hline
\end{tabular}

not necessarily equal to $v$, differences between $w$ and $w_{e}$ are expected, whereas estimates of $\left|\Gamma_{o}\right|$ are expected to be accurate. Using these estimates with (4) leads to the results shown in Fig. 11 as solid black lines, in close agreement with experimental results. Average residual errors between measurements and (4) were smaller than $2 \%$ of $\left|\Gamma_{o}\right|$.

The fault parameters thus obtained are shown in Table I and will be used throughout this section as references. Other parameters pertaining to the faults are presented in Table I, such as the distance $d_{o}$ where the fault starts and its length $w$. A uniformly distributed uncertainty spanning a $\pm 0.5 \mathrm{~mm}$ range is assumed ${ }^{1}$ for these geometrical distances, due to $1 \mathrm{~mm}$ resolution of the rigid meter used during the measurements. Faults involving a transition like in the two crushed cables had their length measured from center to center of the two transitions. From these parameters, the critical and characteristic frequencies were estimated and shown in the same table.

${ }^{1}$ i.e., a type $B$ evaluation of uncertainty.

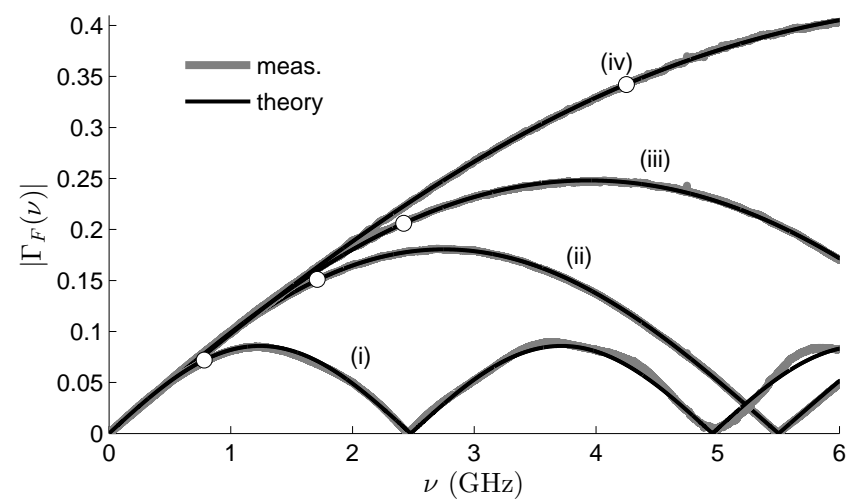

Figure 11. Frequency-domain reflection coefficients of the four faults in Fig. 9, comparing experimental results (thick background gray curves) and theoretical description from (4) once the fault parameters are identified (black curves). White circles mark the critical frequency $f_{o}$ for each fault, below which their responses are well approximated by a linear function.
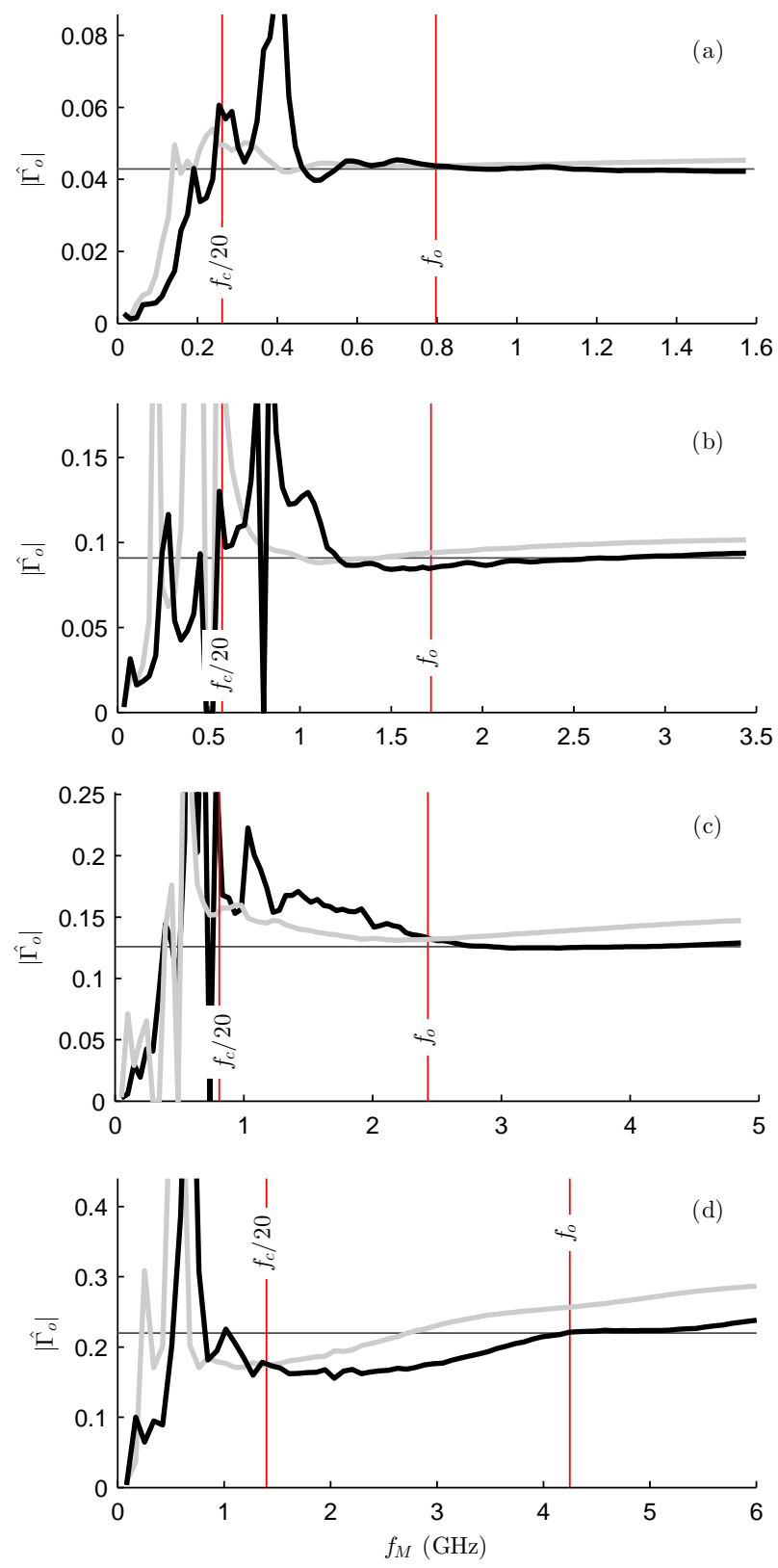

Figure 12. Estimates of fault severity $\left|\hat{\Gamma}_{o}\right|$, obtained from a polynomial fit of order $N=1$ (gray lines) and $N=2$ (black lines), as functions of the test bandwidth $f_{M}$, going from $10 \mathrm{MHz}$ up to $2 f_{o}$ for each fault, but for case (iv), where $2 f_{o}>6 \mathrm{GHz}$. Thin horizontal lines reproduce the reference values for the severity found in Table I. Plots (a) to (d) correspond to the four faults in Fig. 9, respectively from (i) to (iv).

\section{A. Fault identification}

The identification procedure detailed in Sec. II-A was applied to the data shown in Fig. 11. Only the amplitude $\left|\Gamma_{\text {in }}(\nu)\right|$ was used, selecting only samples below $f_{M}$, which was made to vary over the frequency range $10 \mathrm{MHz}$ to $2 f_{o}$, having used the $f_{o}$ estimates in Table I for each of the four faults. Tests were not carried out at higher $f_{M}$, since $f_{c} / 4 \simeq f_{o} \pi / 2<2 f_{o}$ and that data at $f_{c} / 4$ are sufficient to identify the fault directly [14].

The results of the identification procedure were compared 

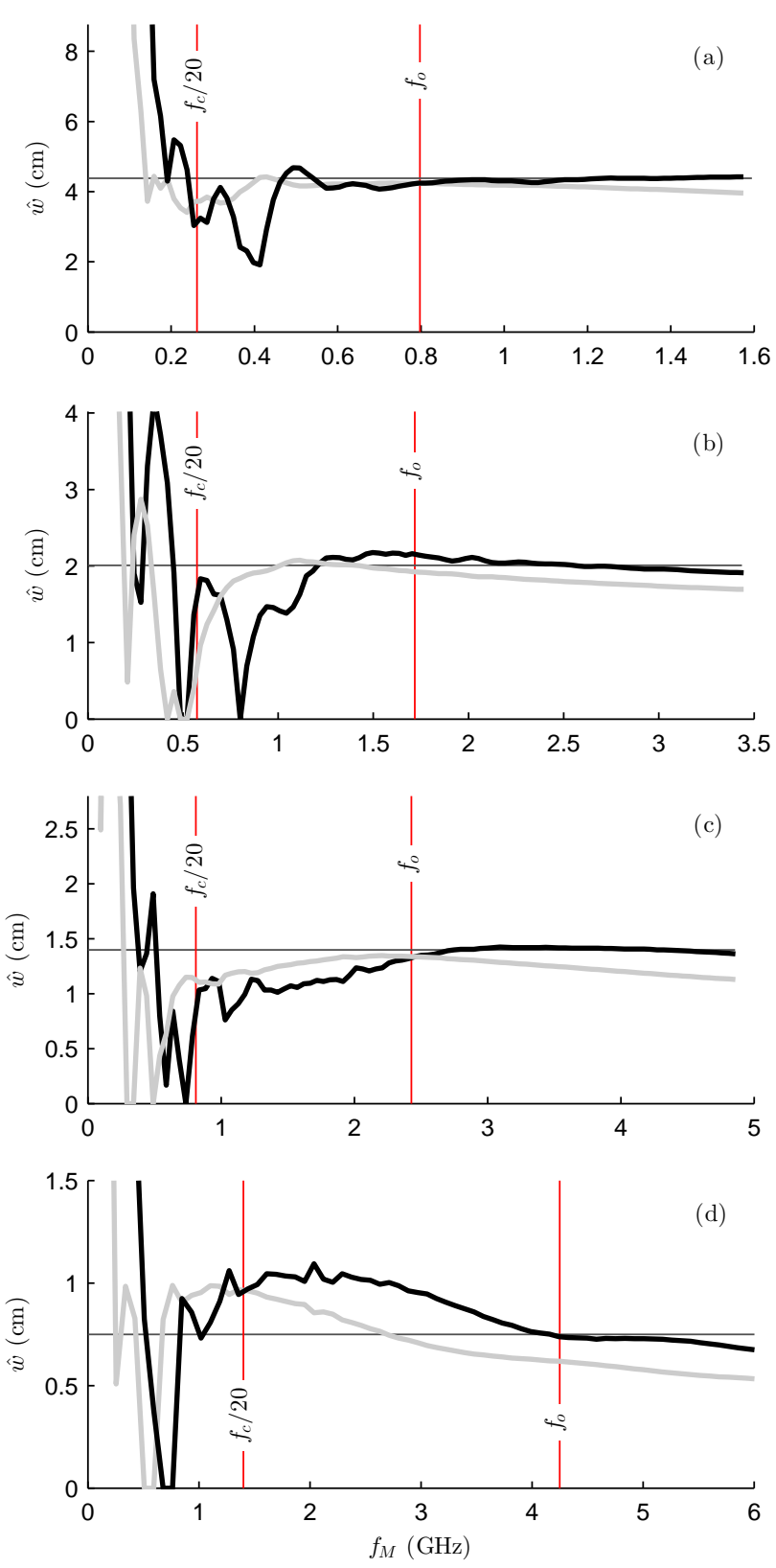

Figure 13. Same as in Fig. 12, but results here pertain to estimates of the fault length $w$.

for the case of polynomial fits of order $N=1$ and $N=2$, in order to verify the validity of the analysis in Sec. II-B, where criteria for choosing the order of the polynomial fit were presented.

Results for this analysis are shown in Figs. 12 and 13, together with the fault parameters estimated from high-frequency samples presented in Table I, serving as references. The choice of the polynomial fit has a direct impact on the results, with $N=1$ providing the best results for $f_{M}<f_{o}$, while $N=2$ performs better at higher frequencies. The analysis in Sec. II-B predicted that for $N=1$ the estimates of the fault parameters should be precise up to about $5 \%$ for $\left|\Gamma_{o}\right|=0.1$ which is consistent with estimates around $f_{o}$ for the first three faults,

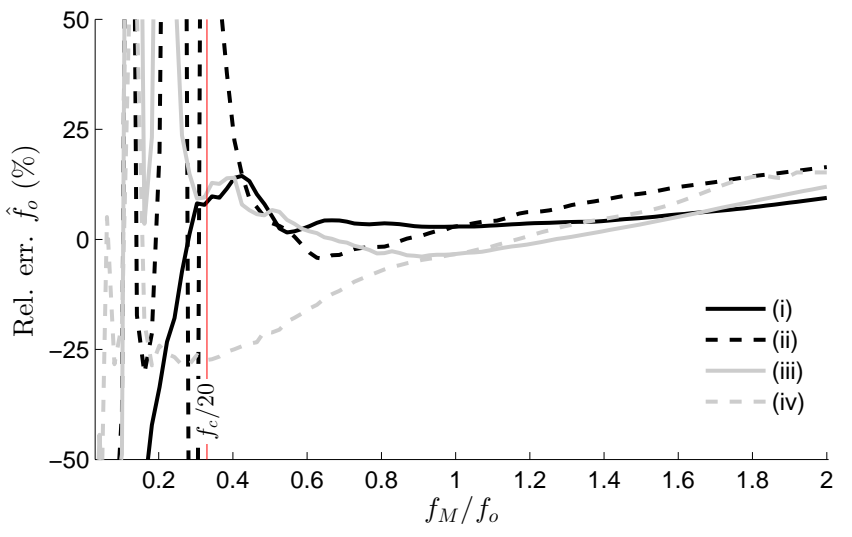

Figure 14. Estimates of the critical frequency $f_{o}$ for the four faults, based on (23) for a polynomial fit with $N=1$, as a function of the test bandwidth $f_{M}$.

while for the fourth one, which is more severe, is expected to be closer to the $36 \%$ error predicted for $\left|\Gamma_{o}\right|=0.3$. As $N=2$, the estimators error should be smaller than $5 \%$ of the real parameters, consistently with all of the faults, apart for the most severe, for which $N=3$ should be used approaching the frequency $2 f_{o}$.

At frequencies below about $f_{o} / 3 \simeq f_{c} / 20$ all estimates start to fluctuate. These errors are caused by the accrued sensitivity of the least-square fit (10) to even minor deviations between the theoretical model (4) and the experimental results, e.g., due to noise but also limitations of the model (4) which neglects border effects and transitions at the ends of the faults.

This kind of behavior is consistent with the analysis in Sec. II-B, where a minimum $f_{M}>f_{\alpha}$ was discussed as necessary in order to ensure a robust least-square fit. The fact that estimates break down below $f_{c} / 20$ can be compared with the results in Fig. 5, inferring that the ratio $p_{1} / p_{0}$ of the polynomial coefficients for the experimental dataset is subject to variations close to $2 \%$ from the theoretical model. The same figure predicts that for more severe faults the estimator should behave better at lower frequency, as the curvature of $\left|\Gamma_{F}(\nu)\right|$ is more pronounced, thus easier to identify. The results in Figs. 12 and 13 for the case (iv) support this last prediction, with a region free of fluctuations extending to lower frequencies.

The pertinence of introducing a minimum frequency $f_{\alpha}$ is confirmed, making it fundamental to know if any attempt to identifying a fault parameters is carried out over a sufficiently wide frequency range. For this reason the $f_{o}$ estimator (23) was also tested, with results reported in Fig. 14. Estimates of $f_{o}$ were found to be precise to within $\pm 20 \%$ for $f_{M}>0.5 f_{o}$ for the four faults tested, while at lower frequencies rapid fluctuations are observed. A simple criterion ensues, where the estimate of $f_{o}$ can be expected to be reliable only when weakly affected by a changing $f_{M}$.

\section{B. Standard TDR echoes}

This section explores the issue of how TDR echoes can be ambiguous if interpreted under the usual paradigm applied to hard faults, i.e., that their intensity is a measure of how severe 
a fault is. Two sets of signals were considered: those observed with standard TDR and alternatively those obtained with the equalization approach presented in Sec. II-C.

The test signal $p(t)$ was chosen to be a base-band pulse with a Fourier spectrum $P(\nu)$ given by a Kaiser window [21], spanning a bandwidth $f_{M}$. The rationale for this choice was driven by the ability of Kaiser windows to approximate Slepian functions, which in turn are the optimal solution for maximal energy concentration, or minimum time-span, of $p(t)$, for a given bandwidth $f_{M}$. A taper parameter $\beta=5$ was used, approximatively corresponding to a $30 \mathrm{~dB}$ taper in the frequency domain and a $36 \mathrm{~dB}$ side-lobe level in time domain, in order to reduce the appearance of side lobes while minimizing loss of temporal and spatial resolution. While this choice is guided by the need for a highly resolved pulse, any pulse shape is ultimately subject to the uncertainty principle intrinsical to Fourier transform [22], implying that for a bandwidth $f_{M}$, a pulse would occupy a spatial interval at best equal to $v / f_{M}$. In other words, a fault of length $w$ could be resolved only for $f_{M} \gtrsim f_{c} / 2$.

The TDR echoes $e(t)$ were computed from the input reflection coefficient $\Gamma_{\text {in }}(\nu)$ of each faulty cable, by inversetransforming $\Gamma_{\text {in }}(\nu) P(\nu)$, having taken care to normalize $P(\nu)$ such that $p(t)$ peaked to one, in order to simplify any comparison of results. Results are presented in Fig. 15, for test signals extending over three different bandwidths $f_{M}$. Fig. 15(a) shows TDR echoes for $f_{M}=1 \mathrm{GHz}$, for which all four faults are tested well below their critical frequency $f_{o}$, apart for case (i). The resulting echoes are very similar, apart for a changing sign between cases (i)-(ii) and (iii)-(iv). As pointed out in [14], such TDR signals would be associated to faults of similar severity, even though $\left|\Gamma_{o}\right|$ goes from 0.042 up to 0.22 . Switching to $f_{M}=3 \mathrm{GHz}$ partially removes this ambiguity, as shown in Fig. 15(b), where the echo from fault (i) has peaked to its respective $\left|\Gamma_{o}\right|$, while the remaining echoes still display similar amplitudes. Using the full bandwidth $f_{M}=6 \mathrm{GHz}$ results in more insightful results in Fig. 15(c), with case (i) now clearly featuring two separate echoes from the ends of the faulty section, as well as cases (ii) and (iii), even though the two echoes are not yet fully separated for these last two cases. The echo from fault (iv) only peaks at 0.13 , thus making it unlikely to suspect a much more severe impedance mismatch of 0.22 .

Fig. 15(c) suggests that standard TDR, if applied to a sufficiently wide bandwidth, can still be used for assessing a impedance fault. In order to understand under what conditions this approach is feasible, two further estimators were tested: the fault severity was assessed by measuring $\max _{t}|e(t)|$ and the fault length from the time delay between the two peaks observed in standard TDR echoes, corresponding to a distance $w_{p}$. Fig. 16 shows how these estimators fare for a changing test bandwidth. The results for the four faults are thoroughly consistent and superpose to provide a continuum.

Therefore, a naive interpretation of TDR echoes can work only for $f_{M}>f_{c} / 2$. Yet, it is important to stress how unlikely it is to have the opportunity of testing faults at such high frequencies, with Fig. 15(a) a likelier outcome that serves as a sobering remainder of the ambiguities of interpreting TDR
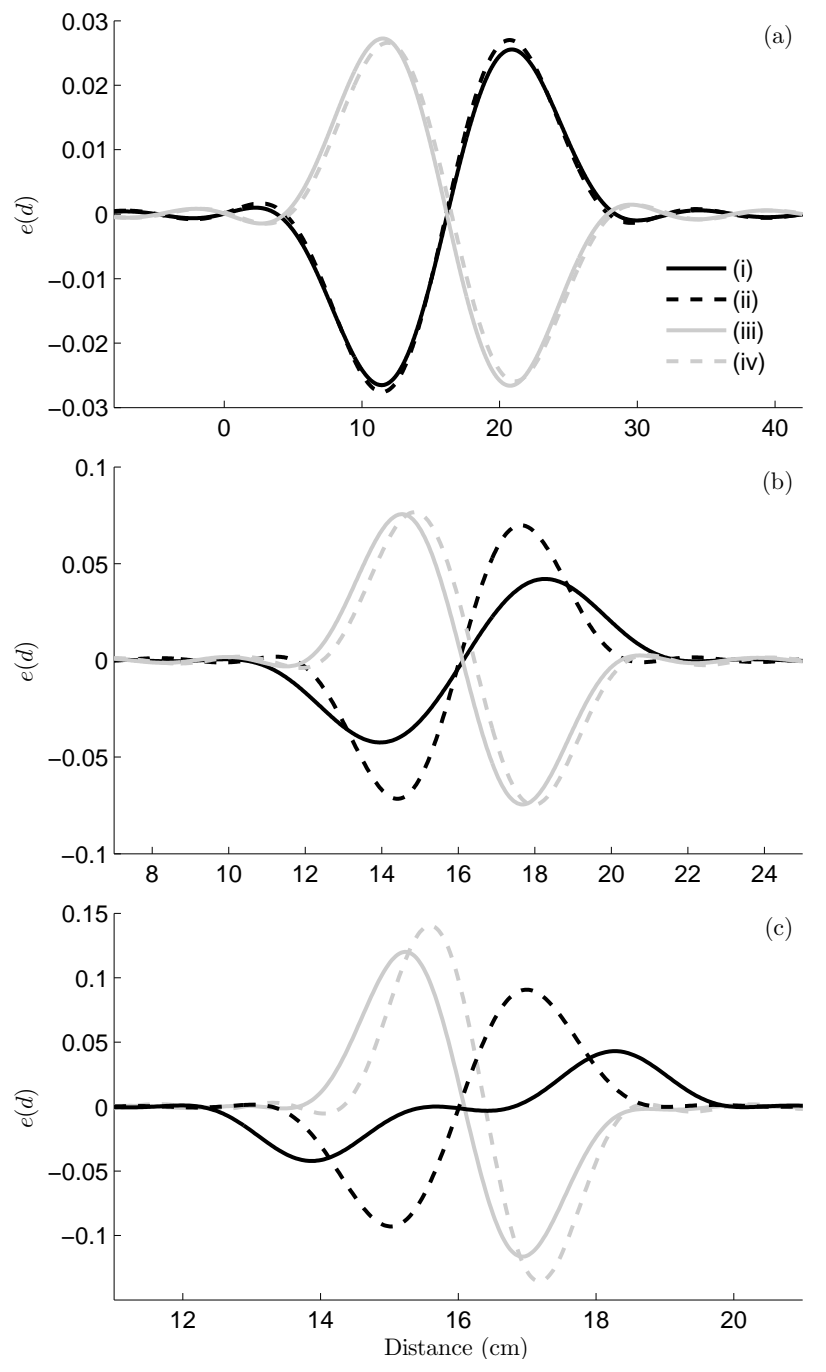

Figure 15. Standard TDR echoes for the four faults tested with base-band pulses $p(t)$ with a bandwidth $f_{M}$ : (a) $1 \mathrm{GHz}$, (b) $3 \mathrm{GHz}$ and (c) $6 \mathrm{GHz}$. The reflectograms are expressed in terms of the distance from the test port.
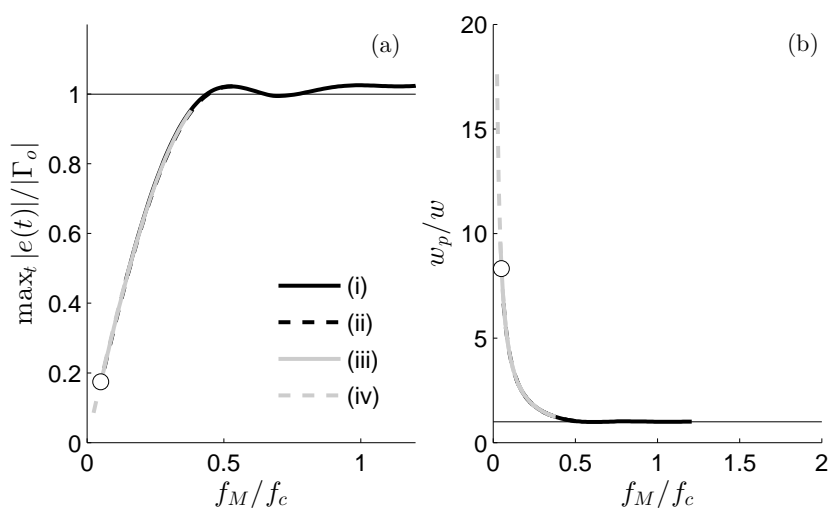

Figure 16. Estimates of fault parameters obtained by direct inspection (peak reflection and peak spacing) of standard TDR echoes for $f_{M} \in[0.2,6] \mathrm{GHz}$. Results for the four faults are indistinguishable and create a continuum. The white circles indicate $f_{c} / 20$, i.e., the lower limit for a reliable identification with the proposed procedure. The thin horizontal lines stand for exact estimates. 

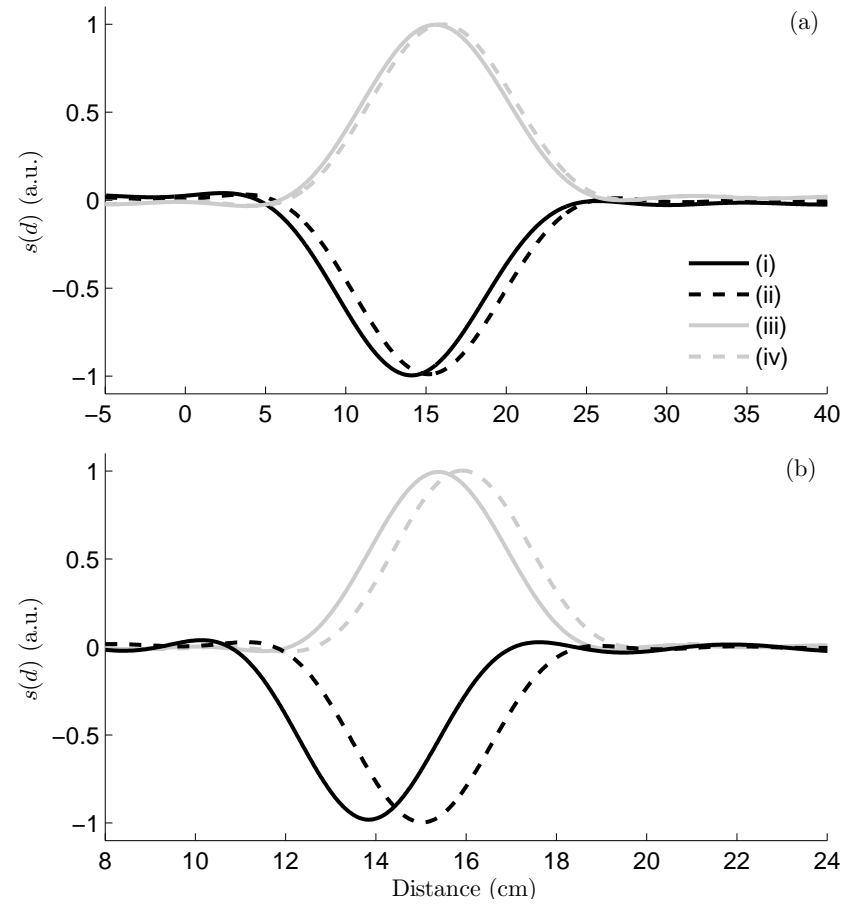

Figure 17. Synthetic TDR echoes obtained after the equalization (25), for a bandwidth $f_{M}$ : (a) $1 \mathrm{GHz}$ and (b) $3 \mathrm{GHz}$. Results should be compared with those in Fig. 15(a)-(b).

echoes in the case of soft faults. Results in Fig. 16 are in sharp contrast with those in Figs. 12 and 13, and prove the effectiveness of the procedure presented in Sec. II-B, where data collected for $f_{M}=1 \mathrm{GHz}$ were largely sufficient to precisely estimate the fault parameters within a few percent points of the reference values.

\section{Equalized TDR echoes}

When applying the equalization procedure (25) presented in Sec. II-C fault echoes are transformed into those shown in Fig. 17, obtained by using the fault parameters estimated in Figs. 12 and 13 with polynomial fits with : $N=1$ for $f_{M}=1 \mathrm{GHz}$, in Fig. 17(a) and with $N=2$ for $f_{M}=3$ GHz in Fig. 17(b), respectively. Three main effects can be noticed: first, the disappearance of the monocycle shape, with echoes $s(t)$ now proportional to the original test signal $p(t)$, as expected for hard faults. Second, the echoes display a peak that is positioned at the left end of the faulty section of a cable. Finally, the polarity, or sign, of $\Gamma_{o}$ is clearly apparent, as demonstrated by the results in Fig. 17. The fact that the peak amplitude of the echoes after equalization be equal to one is a by-product of (25) and a gauge of the accuracy of the fault identification.

As argued in the previous sections, standard TDR testing of faults below their critical frequency $f_{o}$ not only yields ambiguous results, as the fault severity cannot be estimated, but also the exact position of the fault is not directly apparent.

The accuracy offered by the equalization procedure can be assessed from the results in Fig. 17. The distance $\hat{d}_{o}$ for which their peaks are observed is reported in Table II, and the
Table II

FAULT LOCATION USING EQUALIZATION: ESTIMATES OF THE LEFT-END POSITION OF THE FAULTY SECTION $d_{o}$ IN CASE THE FAULT PARAMETERS ARE IDENTIFIED AND OF THE DISTANCE TO THE FAULT CENTER $d_{c}$ WHEN NO IDENTIFICATION IS APPLIED, FOR $f_{M}=1 \mathrm{GHZ}$ (STANDARD TYPEFACE) AND $f_{M}=3 \mathrm{GHZ}$ (ITALICS). ERRORS ARE EVALUATED WITH RESPECT TO THE REFERENCE RESULTS IN TABLE I.

\begin{tabular}{|c|c|c|c|c|}
\hline \multirow{2}{*}{ Faults } & $\hat{d}_{o}$ & $\hat{d}_{o}-d_{o}$ & $\hat{d}_{c}$ & $\hat{d}_{c}-d_{c}$ \\
\cline { 2 - 5 } & $(\mathrm{cm})$ & $(\mathrm{mm})$ & $(\mathrm{cm})$ & $(\mathrm{mm})$ \\
\hline \hline \multirow{2}{*}{ (i) } & 14.1 & 2 & 16.2 & 1 \\
& 13.9 & 0 & 16.1 & 0 \\
\hline \multirow{2}{*}{ (ii) } & 15.2 & 2 & 16.1 & 1 \\
& 15.0 & 0 & 16.0 & 0 \\
\hline \multirow{2}{*}{ (iii) } & 15.6 & 2 & 16.2 & 2 \\
& 15.4 & 0 & 16.1 & 1 \\
\hline \multirow{2}{*}{ (iv) } & 16.0 & 0 & 16.5 & 1 \\
& 15.9 & -1 & 16.4 & 0 \\
\hline
\end{tabular}

Table III

VERY-LOW FREQUENCY $\left(f_{M} / f_{c} \ll 1\right)$ FAULT LOCATION USING EQUALIZATION WITHOUT FAULT IDENTIFICATION, BASED ON POLYNOMIAL FITS OF ORDER $N=1$.

\begin{tabular}{|c|c|c|c|c|c|c|}
\cline { 2 - 7 } \multicolumn{1}{c|}{} & \multicolumn{3}{c|}{$f_{M}=0.2 \mathrm{GHz}$} & \multicolumn{3}{c|}{$f_{M}=0.5 \mathrm{GHz}$} \\
\hline \multirow{2}{*}{ Faults } & $d_{e}$ & $d_{e}-d_{c}$ & $f_{M} / f_{c}$ & $d_{e}$ & $d_{e}-d_{c}$ & $f_{M} / f_{c}$ \\
\cline { 2 - 7 } & $(\mathrm{cm})$ & $(\mathrm{mm})$ & $\%$ & $(\mathrm{~cm})$ & $(\mathrm{mm})$ & $\%$ \\
\hline \hline (i) & 17.5 & 14 & 4.0 & 16.4 & 3 & 10 \\
(ii) & 16.6 & 6 & 1.9 & 16.2 & 2 & 4.8 \\
(iii) & 17.2 & 12 & 1.3 & 16.4 & 4 & 3.2 \\
(iv) & 17.4 & 10 & 0.60 & 16.7 & 3 & 1.4 \\
\hline
\end{tabular}

error with respect to the geometrical distance $d_{o}$ where the beginning of faulty section was measured. Errors are smaller than $2 \mathrm{~mm}$ when testing with $f_{M}=1 \mathrm{GHz}$, and drop below $1 \mathrm{~mm}$ for $f_{M}=3 \mathrm{GHz}$. Geometrical distances are expected to come with an uncertainty of about $\pm 0.5 \mathrm{~mm}$. These values of $f_{M}$ should be compared with the minimum test bandwidth $f_{c} / 2$ required with TDR, as estimated in Table I.

If faults are tested for $f_{M} / f_{c} \ll 1$ where fault identification may not work, an equalization can still be applied, by using the polynomial fit (10) in (25) instead of the fault model (4). Echoes similar to those expected for a full equalization are expected, but they would now reach their peaks for $t=T+\tau$, corresponding to a distance $d_{e}$ expected to be equal to the distance $d_{c}$ at which the center of the fault is found, rather than its left end. Results obtained with this procedure are reported in Table III, where the four faults were tested at $f_{M}=0.2$ and $0.5 \mathrm{GHz}$, were their parameters cannot be reliably estimated. This equalization is still rather accurate, and results in errors about $1 \mathrm{~cm}$ for $f_{M}=0.2 \mathrm{GHz}$, and $3 \mathrm{~mm}$ for $f_{M}=0.5 \mathrm{GHz}$.

\section{CONClusions}

This paper has introduced an identification approach capable of determining the nature of impedance faults along a cable. It has the remarkable advantage of allowing a fault identification without needing to address simultaneously the problem of locating it, which is otherwise known to lead to an illposed estimation problem. The factorization is made possible by the fact that the proposed approach only uses amplitude 
information in the frequency domain, which are practically independent from the position of the fault.

The identification procedure was proven to be effective at frequencies as low as $f_{o} / 3 \simeq f_{c} / 20$, i.e., about $10 \%$ of the bandwidth $f_{c} / 2$ required with standard TDR, a limitation due to the uncertainty principle of Fourier transform. Moreover, it was shown to be capable of accurately estimating the impedance mismatch, or severity, of the fault together with its length, even in cases where standard TDR cannot resolve partial reflections from the tested soft faults. This procedure is well-posed and does not rely on any initial guess. It is easily implemented and numerically light, as it is based on a polynomial regression of low-order. An equalization procedure can then be applied in order to remove the ambiguities intrinsical to standard TDR signals for this class of faults.

Although the identification assumes a lossless propagation along the cable, losses can be accommodated by using an iterative approach, compensating most of them by first roughly estimating the distance to the fault from the time-of-flight, proceeding to a first identification of the fault that will subsequently be used in order to provide a better estimate of the fault distance. Such procedure could then be iterated, if needed. Future work will focus on the case of lossy and dispersive cables.

Minimum conditions that ensure the feasibility of the proposed procedure were derived, explaining the reasons for the difficulties in detecting and interpreting this class of faults. These results are expected to pave the way to quantitative methods for designing early-warning testing methods allowing automatic systems to reliably decide whether echoes may be caused by severe impedance faults, before they develop into hard faults, even when they still generate weak echoes.

\section{REFERENCES}

[1] B. M. Oliver, "Time domain reflectometry," Hewlett-Packard Journal, vol. 15 , no. 6, pp. 1-7, 1964.

[2] C. Buccella, M. Feliziani, and G. Manzi, "Detection and localization of defects in shielded cables by time-domain measurements with UWB pulse injection and clean algorithm postprocessing," IEEE Trans. Electromagn. Compat., vol. 46, no. 4, pp. 597 - 605, November 2004.

[3] C. Furse, Y. C. Chung, C. Lo, and P. Pendayala, "A critical comparison of reflectometry methods for location of wiring faults," Smart Structures and Systems, vol. 2, no. 1, pp. 25-46, 2006.

[4] E. Song, Y.-J. Shin, P. Stone, J. Wang, T.-S. Choe, J.-G. Yook, and J. B. Park, "Detection and location of multiple wiring faults via timefrequency-domain reflectometry," IEEE Transactions on Electromagnetic Compatibility, vol. 51, no. 1, pp. $131-138$, feb. 2009.

[5] L. Abboud, A. Cozza, and L. Pichon, "A matched-pulse approach for soft-fault detection in complex wire networks," IEEE Trans. Instrum. Meas., vol. 61, no. 6, pp. 1719-1732, June 2012.

[6] C. K. Lee, K. S. Kwak, T. S. Yoon, and J. B. Park, "Cable fault localization using instantaneous frequency estimation in gaussian-enveloped linear chirp reflectometry," IEEE Trans. Instrum. Meas., vol. 62, no. 1, pp. 129-139, Jan 2013.

[7] S. J. Chang and J. B. Park, "Multiple chirp reflectometry for determination of fault direction and localization in live branched network cables," IEEE Transactions on Instrumentation and Measurement, vol. 66, no. 10, pp. 2606-2614, Oct 2017.

[8] J. Zhang, Y. Zhang, and Y. Guan, "Analysis of time-domain reflectometry combined with wavelet transform for fault detection in aircraft shielded cables," IEEE Sensors Journal, vol. 16, no. 11, pp. 4579-4586, June 2016.
[9] Y. J. Shin, E. J. Powers, T. S. Choe, C.-Y. Hong, E.-S. Song, J.-G. Yook, and J. B. Park, "Application of time-frequency domain reflectometry for detection and localization of a fault on a coaxial cable," IEEE Transactions on Instrumentation and Measurement, vol. 54, no. 6, pp. 2493-2500, Dec 2005.

[10] S. Naik, C. M. Furse, and B. Farhang-Boroujeny, "Multicarrier reflectometry," IEEE Sensors Journal, vol. 6, no. 3, pp. 812-818, June 2006.

[11] L. Griffiths, R. Parakh, C. Furse, and B. Baker, "The invisible fray: A critical analysis of the use of reflectometry for fray location," IEEE Sensors Journal, vol. 6, no. 3, pp. 697-706, June 2006.

[12] S. Schuet, D. Timucin, and K. Wheeler, "A model-based probabilistic inversion framework for characterizing wire fault detection using TDR," IEEE Trans. Instrum. Meas., vol. 60, no. 5, pp. 1654-1663, 2011.

[13] A. Cozza and L. Pichon, "Echo response of faults in transmission lines: Models and limitations to fault detection," IEEE Trans. Microw. Theory Tech., vol. 64, no. 12, pp. 4155-4164, Dec 2016.

[14] A. Cozza, "Never trust a cable bearing echoes: Understanding ambiguities in time-domain reflectometry applied to soft faults in cables," IEEE Trans. Electromagn. Compat., 2018, in press.

[15] L. Jing, W. Wang, Z. Li, and R. Murch, "Detecting impedance and shunt conductance faults in lossy transmission lines," IEEE Transactions on Antennas and Propagation, pp. 1-1, 2018.

[16] W. Wang, L. Jing, Z. Li, and R. D. Murch, "Utilizing the born and rytov inverse scattering approximations for detecting soft faults in lossless transmission lines," IEEE Transactions on Antennas and Propagation, vol. 65, no. 12, pp. 7233-7243, Dec 2017.

[17] Q. Zhang, M. Sorine, and M. Admane, "Inverse scattering for soft fault diagnosis in electric transmission lines," IEEE Transactions on Antennas and Propagation, vol. 59, no. 1, pp. 141-148, Jan 2011.

[18] Q. Shi and O. Kanoun, "Wire fault diagnosis in the frequency domain by impedance spectroscopy," IEEE Trans. Instrum. Meas., vol. 64, no. 8 , pp. 2179-2187, Aug 2015 .

[19] R. E. Collin, Foundations for microwave engineering. McGraw-Hill, 1966.

[20] T. P. Ryan, Modern regression methods. John Wiley \& Sons, 2008, vol. 655 .

[21] J. Kaiser and R. Schafer, "On the use of the $I_{0}$-sinh window for spectrum analysis," IEEE Trans. Acoust., Speech, Signal Process., vol. 28, no. 1, pp. 105-107, 1980 .

[22] K. B. Howell, Principles of Fourier analysis. CRC Press, 2001. 